\title{
A Influência da Confiança e da Participação no Orçamento em Uma Instituição De Saúde
}

\section{The Influence of Trust and Participation in The Budget in a Health Institution}

\author{
Loriberto Starosky Filho ${ }^{1}$, Dirceu Rodrigues Dias ${ }^{2}$, Carlos Eduardo Facin Lavarda ${ }^{3}$
}

\begin{abstract}
${ }^{12}$ Programa de Pós-Graduação em Ciências Contábeis da Universidade Regional de Blumenau (FURB), Brasil, ${ }^{3}$ Departamento de Ciências Contábeis, Universidade Regional de Blumenau, Blumenau, Brasil Correspondência: Loriberto Starosky Filho, Orplan Consultoria, Endereço: Rua Hugo Loth Júnior 167, CEP.: 89.056-450 Blumenau, Brasil. Tel: 5547 3323-7950. E-mail: starosky@ terra.com.br
\end{abstract}

Recebido: 30 de Março de 2012 Aceito: 07 de Julho de 2015 Publicado: 01 de Dezembro de 2015

\begin{abstract}
Resumo
O artigo investiga a avaliação de comportamento de gestores, a ênfase ao orçamento, a confiança, a participação orçamentária e a tensão relacionada ao trabalho, aplicada numa instituição filantrópica de saúde. O objetivo foi identificar qual a influência que a confiança, a ênfase e a participação no orçamento têm sobre a tensão relacionada ao trabalho em uma instituição de saúde. O estudo tem característica quantitativa, com a utilização de métodos estatísticos para mensuração das variáveis. A técnica de análise de dados utilizada foi a mesma do artigo base de Lau e Buckland (2001), ou seja, a análise de caminho, com uso de estatística descritiva e correlações para testar e confirmar as seguintes hipóteses: H1: a ênfase no orçamento tem um efeito indireto sobre a tensão relacionada ao trabalho através da confiança; H2: a ênfase no orçamento tem um efeito indireto sobre a tensão relacionada ao trabalho através da participação orçamentária; H3: a participação orçamentária tem um efeito indireto sobre a tensão relacionada ao trabalho através da confiança.
\end{abstract}

Palavras-chave: Participação orçamentária, ênfase no orçamento, confiança.

\section{Abstract}

The article investigates the behavioral assessment of managers, budget emphasis, trust, budget participation and job related tension, applied in a philanthropic health organization. The aim was to identify what influence trust, emphasis and budget participation have on the job related tension in a philanthropic health organization. The study is quantitative trait, with the use of statistical methods for measurement of the variables. The data analysis technique used was the same as the basis of Article Lau and Buckland (2001), ie, the path analysis, using descriptive statistics and correlations to test and confirm the following hypotheses: H1: the emphasis in the budget have an indirect effect on job-related tension through trust; $\mathrm{H} 2$ : the emphasis in the budget has an indirect effect on the stress related to work through the budget share; H3: budget participation has an indirect effect on work-related stress through trust.

Keywords: Budget participation, budget emphasis, Trust.

Esta obra está licenciada sob uma Licença Creative Commons Attribution 3.0.

\section{Introdução}

No âmbito da contabilidade gerencial, várias pesquisas foram desenvolvidas para entender os impactos que os estilos de avaliação de desempenho de gestores exercem sobre suas equipes. Embora no Brasil estes estudos ainda estejam em fase inicial (NASCIMENTO; RIBEIRO; JUNQUEIRA, 2008), encontram-se diversos estudos sobre o assunto na literatura internacional, sendo o orçamento destacado como um dos métodos de avaliação mais utilizados.

O orçamento tem sido utilizado pelas organizações para diversas finalidades. Nascimento (2006) destaca os seguintes objetivos do orçamento: a maximização e a utilização dos fatores ambientais internos e externos que afetam a organização; a avaliação e a adequação de seus planos; a base para avaliação de desempenho gerencial; a promoção da coordenação e comunicação gerencial; e o apoio a precificação. Além de mensurar os fatores de 
desempenho financeiro, ele pode ser utilizado também para avaliar o comportamento de gestores e equipes.

O presente estudo investiga a avaliação de comportamento de gestores, a ênfase ao orçamento, confiança, a participação orçamentária e a tensão relacionada ao trabalho, aplicada numa instituição filantrópica de saúde. Utiliza como base a abordagem apresentada por Lau e Buckland (2001), que em seu estudo verificaram as influências da confiança e participação orçamentária na tensão relacionada ao trabalho, estudo este realizado com gestores na Noruega.

Assim, o objetivo do presente estudo é identificar qual a influência que a confiança, a ênfase e a participação no orçamento têm sobre a tensão relacionada ao trabalho em uma instituição de saúde.

Para atingir o objetivo, o estudo irá testar as seguintes hipóteses: H1: a ênfase no orçamento tem um efeito indireto sobre a tensão relacionada ao trabalho através da confiança; H2: a ênfase no orçamento tem um efeito indireto sobre a tensão relacionada ao trabalho através da participação orçamentária; H3: a participação orçamentária tem um efeito indireto sobre a tensão relacionada ao trabalho através da confiança.

Estudos realizados em contabilidade gerencial que focam a abordagem comportamental geralmente são realizados em empresas que visam lucro e são de capital aberto. Poucos são os estudos com esta abordagem realizados em instituições sem fins lucrativos, e mais especificamente na área da saúde. Esta é a principal contribuição deste estudo, inserindo uma organização sem fins lucrativos com atuação na área hospitalar no contexto das pesquisas em contabilidade gerencial.

\section{Desenvolvimento da Teoria}

Nesta seção, trazem-se os posicionamentos teóricos que serviram de base para o desenvolvimento da linha de raciocínio da pesquisa.

\section{1 Ênfase no Orçamento e Tensão Relacionada ao Trabalho}

As organizações fazem uso de medidas de desempenho com o fim de promover um comportamento positivo dos gestores, e entre algumas destas medidas destaca-se a ênfase no orçamento.

O trabalho de Hopwood (1972) é considerado por diversos autores, a exemplo de Ross (1995) e Lau e Buckland (2001), como sendo o primeiro a observar de forma sistemática o comportamento dos subordinados, quando avaliados mediante o uso de dados contábeis do orçamento. Hopwood (1972) ressalta que a avaliação de desempenho tem provocado comportamentos disfuncionais nos subordinados, ou seja, na medida em que eles tomam conhecimento que serão avaliados com base em dados contábeis, procuram tratar estes dados de forma a trazer o melhor resultado para si, superestimando as despesas e subestimando as receitas.

Como consequência, esta medida de avaliação de desempenho pode gerar no avaliado uma tensão relacionada ao trabalho, com consequentes problemas de relacionamento com superiores, com seus pares e também com a manipulação dos dados contábeis (HOPWOOD, 1972).

Segundo Otley (1978), a informação do orçamento usada como base de avaliação de desempenho provavelmente irá determinar como o gestor responderá às informações contábeis, por conta do impacto que isso trará nos resultados da organização. A Figura 1 dimensiona como ocorrem estas diferenças de percepção entre o que a organização espera (planejado) e o que os gestores fazem (realizado).

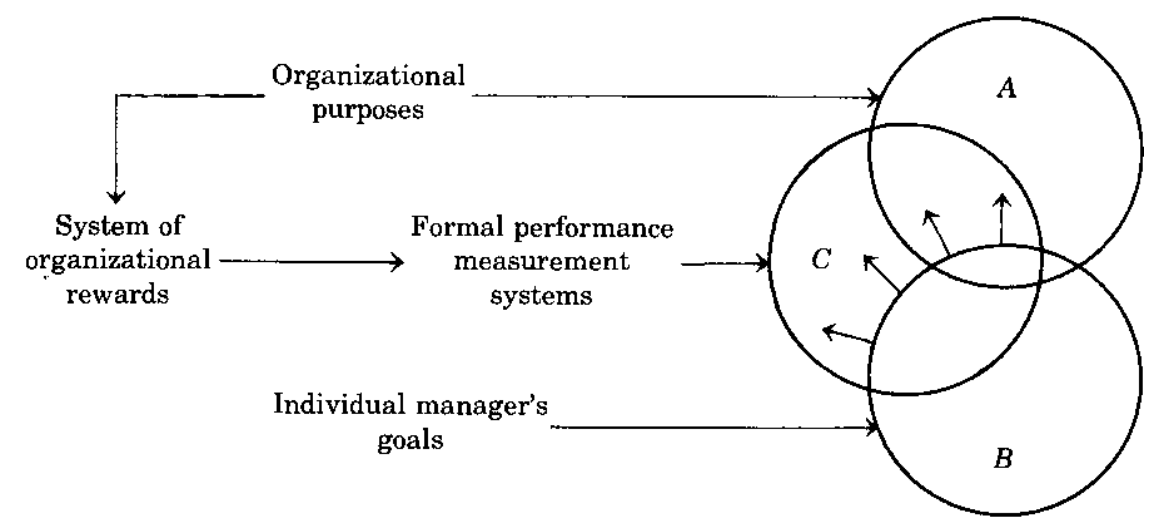

Figura 1: The measurement-reward process with imperfect measurement. $A=$ behavior necessary to achieve organizational purposes; $B=$ behavior actually engaged in by individual manager; $\mathrm{C}=$ behavior formally measured by 
No estudo realizado por Ross (1995), o autor destaca que a tensão relacionada ao trabalho não pode ser generalizada. Estudos posteriores aos de Hopwood e Otley demonstraram que esta variável não apresentava significância em determinados contextos. Conclui Ross (1995) que dependendo do contexto em que a organização está inserida, a tensão é inconsistente com os resultados do estudo de Hopwood.

Apresenta-se para este estudo o modelo proposto por Lau e Buckland (2001), demonstrado na Figura 2, e demonstra que as relações entre a tensão relacionada ao trabalho e as outras variáveis podem ocorrer de forma indireta.

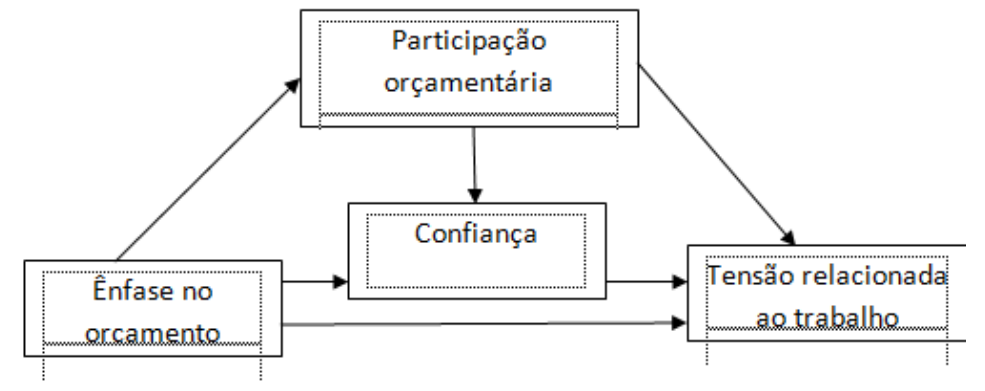

Figura 2: Análise de caminho

Fonte: Adaptado de Lau e Buckland (2001).

\section{2 Ênfase no Orçamento e Confiança}

A confiança entre superior e subordinado é importante para a elaboração, acompanhamento e execução dos orçamentos das organizações. "A confiança entre as pessoas é um dos componentes essenciais do relacionamento interpessoal e da sociabilidade" (NOVELLI, FISCHER, MAZZON, 2006, p.442). "A confiança é definida como sendo aquela relacionada à firme conviç̧ão que o subordinado tem em relação à justiça do julgamento que o superior fará a seu respeito" (LAU; BUCKLAND, 2001).

Hopwood (1972) destaca que sistemas de contabilidade são elaborados para prover todos os níveis de gestão, com informaç̃̃es em tempo oportuno e com razoável grau de precisão, essas informações ajudarão o gestor a decidir ações que irão de encontro aos objetivos da organização. Assim a avaliação de desempenho gerencial, segundo o autor, faz com que os gerentes possam apresentar comportamentos disfuncionais, com tendência a manipular informações para apresentar melhores resultados em sua avaliação de desempenho, o que pode prejudicar os objetivos da organização.

A constituição de reservas orçamentárias que não foram definidas em conjunto com a alta administração é um dos principais problemas. Isso ocorre quando o gestor, sem a autorização de seus superiores, superestima os custos e despesas, subestima as receitas e eleva a demanda de recursos sob sua responsabilidade, desta forma o objetivo do gestor ao compor a reserva, não aceita pela organização, é proteger-se de uma fraca avaliação de desempenho (JUNQUEIRA; OYADAMARI; MORAES, 2010).

Lau e Bukland (2001) investigaram os efeitos da intervenção da confiança e da participação no orçamento e a tensão relacionada ao trabalho. Os resultados apontaram que a ênfase do orçamento tem um efeito insignificante sobre a tensão relacionada ao trabalho, mas um forte efeito indireto por meio da confiança e participação.

Uma das questões levantadas por Lau e Bukland (2001) está relacionada à avaliação de desempenho dos subordinados. Eles dizem que o estilo de avaliação de desempenho escolhido pelos superiores precisa gerar confiança nos subordinados para que estes ajam sem sentir-se constrangidos em expressar as suas opiniões. Destacam ainda que um possível desacordo dos subordinados relacionados ao estilo de avaliação é uma provável fonte de conflitos entre o subordinado, superiores e também outros membros da organização.

Otley (1978) afirma que "Para avaliar o desempenho gerencial, é necessário ter algum tipo de padrão contra o qual a medida de desempenho pode ser avaliada " (OTLEY, 1978, p. 233). O autor destaca que isso compreende considerações de eficácia, se o gerente está fazendo a coisa certa e também de eficiência, se o gerente está fazendo o que ele faz com o menor custo. Neste sentido o autor afirma que "dados orçamentais podem desempenhar um papel importante [...] para representar padrões de eficácia e eficiência” (p.124).

\section{3 Ênfase no Orçamento e Participação Orçamentária}

O envolvimento dos colaboradores no processo de elaboração do orçamento apresenta-se como favorável à uma maior aceitação das metas e objetivos traçados pela administração da organização. "Participação é um conceito 
http://www.revista.ufpe.br/gestaoorg

utilizado para descrever na medida em que um subordinado é permitido para selecionar seus próprios cursos de ação" (MILANI, 1975, p. 274). "Um estilo de liderança focada no orçamento é mais eficaz sob condições de alta participação, mas é ineficaz, onde participação é baixa" ( BROWNELL, 1982, p.1 ).

\subsection{Participação Orçamentária e Tensão Relacionada ao trabalho}

A participação no orçamento também tem sido objeto de estudo de vários pesquisadores, a exemplo da ênfase no orçamento, confiança e tensão relacionada ao trabalho. Joshi, Al-Mudhaki e Bremser (2003) destacam que há resultados conflitantes sobre a real importância da participação no orçamento, na avaliação de desempenho. Os autores citam os trabalhos de Stedry (1960) e Cherrington e Cherrington (1973) com relação negativa entre a participação no orçamento e tensão, enquanto Merchant (1987) e Brownell (1982) relataram uma relação positiva.

No estudo de Hofstede (1983), a participação no orçamento é benéfica, pois contribui para diminuir a distância hierárquica dentro de uma organização. Isto acontece especialmente em sociedades em desenvolvimento.

Esta percepção é verificada em Lau, Low e Eggleton (1997), em seu estudo transcultural analisando comportamentos de gestores na Austrália e em Cingapura. A constatação é de que a participação possui maior influência em sociedades com baixo individualismo e em desenvolvimento.

Para Nouri e Parker (1998), a participação no orçamento beneficia os subordinados em várias maneiras. Em primeiro lugar permite-lhes entender o processo de fixação de metas de orçamento melhor e em segundo lugar a garantia de adequação do orçamento, pois os subordinados podem buscar informações e trocar idéias com os superiores sobre a conclusão das tarefas.

Outro enfoque é o sugerido no estudo de Brownell e Hirst (1986, p.242), em que uma participação elevada no orçamento da organização permite aos subordinados, entre outras coisas "obter acesso a recursos que podem ser usados para amortecer o desempenho da tarefa dos efeitos imprevistos dos outros, e para introduzir novos e melhores meios de resolver as tarefas, que, se altamente incertos, terão características que mudam com o tempo".

Apesar dos estudos sugerirem que a participação no orçamento acaba promovendo a coesão do grupo e a partilha de informações, e desta forma contribui para diminuir a tensão relacionada ao trabalho, e que os benefícios tendem a reduzir a ansiedade enfrentada pelos subordinados, não é a visão de Lau e Buckland (2001). Para os autores, a participação no orçamento tem alta probabilidade de estar associada a tensão ao trabalho dos subordinados inferiores. Dito de outra forma, a ênfase do orçamento está prevista para ser positivamente relacionada com a participação no orçamento e a participação no orçamento, por sua vez, está prevista para ser negativamente relacionada à tensão relacionada ao trabalho.

\subsection{Participação Orçamentária e Confiança}

De acordo com Zand (1972) se o superior percebe que não pode contar com a ajuda do colaborador para estipular as metas e acordos vai tentar impor controles em seu comportamento. Isso segundo o autor gera resistência, pois o colaborador percebe a tentativa de controle de comportamento imposta.

Para Zand (1972) “Todo este comportamento, seguindo a partir de uma falta de confiança, será prejudicial para a troca de informações, de reciprocidade de influência, e para o exercício do auto-controle, e vai diminuir a eficácia da articulação de resolução de problemas esforços." (ZAND, 1972, p.3).

Para Lau e Buckland (2001) a avaliação de desempenho é geralmente utilizada para motivar os colaboradores para melhorar o seu rendimento. Por esta razão, segundo os autores a avaliação de desempenho é geralmente associada aos sistemas de gratificação com premiação que dependem de seus superiores. Na sequência, apresenta-se a metodologia utilizada no artigo base, de Lau e Buckland (2001).

\subsection{Hipóteses Adotadas no Estudo de Lau e Buckland (2001)}

O estudo realizado por Lau e Buckland (2001) foi publicado na ABACUS, com o objetivo de testar as seguintes hipóteses:

H1: a ênfase no orçamento tem um efeito indireto sobre a tensão relacionada ao trabalho através da confiança;

H2: a ênfase no orçamento tem um efeito indireto sobre a tensão relacionada ao trabalho através da participação orçamentária;

H3: a participação orçamentária tem um efeito indireto sobre a tensão relacionada ao trabalho através da 
confiança.

Os autores justificaram a importância do estudo pela inclusão da variável confiança, que media a relações entre a relação entre a participação e atitudes dos subordinados. Até aquele momento os estudos anteriores não abordaram esta variável.

A amostra da pesquisa foi selecionada aleatoriamente a partir de Kompass Noruega 1998. Foram selecionadas cem organizações, com mais de cem funcionários, e que o uso do orçamento era comum. De cada organização, três gestores foram selecionados para responder ao questionário, totalizando 300 questionários.

Do total de questionários enviados, com envelope de resposta pré-pago, e após um trabalho de follow-up por telefone, retornaram 132 questionários. Destes, 12 foram excluídos por conter falhas no preenchimento, restando para a análise 120 questionários.

Para medição dos resultados, os autores utilizaram-se:

a) Para ênfase no orçamento: uma versão modificada de Hopwwod (1972);

b) Para participação orçamentária: foram utilizados seis itens de Milani (1975);

c) Para confiança: quatro itens de sete pontos de Zand (1972); e

d) Para tensão relacionada ao trabalho, o índice de tensão relacionada ao trabalho, conforme Kahn et al (1964).

O método de análise utilizado na pesquisa de Lau e Buckland (2001) para a verificação dos resultados foi análise de caminho, com uso de estatística descritiva e correlações. Os autores justificam o uso da técnica de análise de caminho por ser amplamente utilizada em pesquisas de contabilidade gerencial, e por ser a mais indicada para avaliar os efeitos das variáveis da pesquisa.

\section{Métodos e Procedimentos da Pesquisa}

A presente pesquisa caracteriza-se como sendo descritiva quanto aos seus objetivos, pois procura estabelecer as relações entre as variáveis utilizadas (GIL, 2006). Segundo Raupp e Beuren (2003, p.81), "a pesquisa descritiva configura-se como um estudo intermediário entre a pesquisa exploratória e a explicativa, ou seja, não é tão preliminar como a primeira nem tão aprofundada como a segunda." Ela procura identificar, relatar e comparar aspectos da pesquisa.

Em relação aos procedimentos, a pesquisa se caracteriza como levantamento, pois busca informações diretamente no grupo cujo comportamento se deseja conhecer. Para Gil (2006) as principais vantagens dos levantamentos são o conhecimento direto da realidade, a economia e rapidez e a quantificação dos dados.

O instrumento utilizado para o levantamento dos dados é um questionário contendo 26 perguntas que foram elaboradas utilizando como base as pesquisas realizados por Hopwood (1972), Milani (1975), Zand (1972) e Fischer e Novelli (2008).

Por fim, no que diz respeito à abordagem utilizada, a pesquisa tem característica quantitativa. De acordo com Raupp e Beuren (2003, p. 92), "a abordagem quantitativa caracteriza-se pelo emprego de instrumentos estatísticos, tanto na coleta quanto no tratamento dos dados."

Nesta pesquisa, utilizar-se-á a mesma técnica de análise de dados do artigo base de Lau e Buckland (2001), ou seja, a análise de caminho, com uso de estatística descritiva e correlações para testar as hipóteses: H1: a ênfase no orçamento tem um efeito indireto sobre a tensão relacionada ao trabalho através da confiança; H2: a ênfase no orçamento tem um efeito indireto sobre a tensão relacionada ao trabalho através da participação orçamentária; H3: a participação orçamentária tem um efeito indireto sobre a tensão relacionada ao trabalho através da confiança.

A análise de caminhos, segundo Hair et al (2005) é o "método que empresa correlações bivariadas simples para estimar as relações em um sistema de equações estruturais, baseado na especificação das relações em uma série de equações de regressão." Desta forma, é possível retratar em um diagrama de caminhos qual o efeito sofrido de cada variável da equação.

Para medir as relações entre as variáveis, foram utilizados os softwares estatísticos SPSS, LHSTAT e LISREL, para o cálculo das regressões, correlações e determinação dos coeficientes de caminho para posterior análise dos resultados. 


\subsection{Instrumentos de Medição das variáveis}

\subsection{1 Ênfase no orçamento}

Para mensurar a ênfase no orçamento foram elaboradas questões que se utilizaram dos conceitos do instrumento desenvolvido por Hopwood (1972). Foram elaboradas, portanto, 5 questões, utilizando uma escala de Lickert de 7 pontos, sendo que ' 1 ' representava discordância total, e '7' concordância total.

As questões procuraram medir a percepção dos gestores em relação à avaliação de seu desempenho, onde itens como "preocupação com custos", "cumprimento de metas", e "senso de justiça com o cumprimento de orçamento" buscavam retratar a dependência de curto e longo prazos nas medidas de desempenho.

\subsubsection{Participação orçamentária}

A participação orçamentária foi medida a partir do instrumento de seis itens desenvolvido por Milani (1975). Da mesma forma como a variável ênfase, foi utilizada uma escala de Lickert de 7 pontos, sendo que ' 1 ' representava discordância total e '7' concordância total.

Lau e Buckland (2001) destacam que este instrumento já fora utilizado em vários estudos anteriores, e citam (Brownell, 1982; Brownell e Hirst, 1986; Brownell e Dunk, 1991; Harrison, 1992; Lauet al, 1995; Nouri e Parker, 1998).

No presente estudo, atingiu-se alta confiabilidade interna, observando-se o valor atingido de alfa de Cronbach de 0,84. Na análise fatorial, atingiu-se um auto-valor de 3,274, com contribuição no fator 1 de 81,8 por cento.

\subsubsection{Confiança}

Para medir a variável confiança, foi utilizado como base o estudo de Zand (1972), e elaboradas 4 questões, também com a utilização de escala de Lickert de 7 pontos. A confiança entre subordinados e superiores, destacada por Fischer e Novelli (2008), tem como objetivo neutralizar a vulnerabilidade observada entre os subordinados. Portanto não trata apenas de uma questão de personalidade, mas de comportamento.

Atingiu-se neste estudo um valor alpha de Cronbach de 0,83 , com indicação de alta consistência interna para o instrumento. Na análise fatorial, também foi possível verificar que os itens estavam fortemente carregados, com autovalor de 4,091, e contribuição no fator 1 de 81,8 por cento.

\subsubsection{Tensão relacionada ao trabalho}

A tensão relacionada ao trabalho foi medida pelo Índice de tensão relacionada ao trabalho, descrito no trabalho de Ross (1995). O índice tem quinze itens, e uma escala de Lickert de sete pontos.

Os itens têm como objetivo medir os sentimentos do indivíduo em relação ao seu ambiente de trabalho. Segundo Lau e Buckland (2001), este instrumento foi amplamente utilizado em estudos anteriores, e citam: Hopwood (1972), Otley (1978), Bottger e Hirst (1988), Hirst (1983), Brownell e Hirst (1986) e Harrison (1992).

$\mathrm{O}$ valor de alfa de Cronbach obtido foi de 0,63 , indicando consistência interna forte par ao instrumento. Na Análise fatorial dos itens, percebeu-se um autovalor 7,751, e contribuição do fator 1 de 70,47 por cento.

\subsection{Dados da Pesquisa}

A partir do delineamento do objetivo geral, as hipóteses a serem testadas e os procedimentos da pesquisa, o questionário foi encaminhado a 55 coordenadores das três áreas: administrativa, hotelaria e enfermagem de uma instituição hospitalar filantrópica da região da Associação dos Municípios do Médio Vale do Itajaí (AMMVI).

A instituição iniciou sua trajetória em 1909. Durante os primeiros anos, a enfermagem ambulante era praticada em toda a região. Posteriormente foi providenciado um quarto para o tratamento dos doentes, e em 1916, uma ampliação deixou o hospital com dois salões com 20 leitos cada.

Atualmente conta com mais de 26 mil metros quadrados de área construída, pioneiro na implantação de equipamentos e serviços como Raio-X (em 1920), Tomografia Computadorizada (1982), Angiografia Digital (1992) e serviço de Medicina Nuclear (1992).

Em 1973 deu-se início aos trabalhos na Unidade de Terapia Intensiva (UTI). Em 1976 criou-se a Comissão de Controle de Infecção Hospitalar (CCIH) e começou o funcionamento da sala de Hemodiálise. Em 1980 iniciaram-se os primeiros transplantes de órgãos, com o primeiro transplante renal. Os primeiros transplantes cardíacos e de fígado do Estado foram realizados em 2000 e em 2002, respectivamente.

A instituição se caracteriza pela liderança nos serviços de alta complexidade no Estado de Santa Catarina. Pioneirismo, infra-estrutura, atendimento humanizado e competência da equipe médica são marcas dos serviços. 
O questionário, composto por 26 questões, foi elaborado com base nos instrumentos de medição abordados na metodologia, representados da seguinte forma: para participação no orçamento 6 questões; para ênfase no orçamento 5 questões; para confiança 4 questões e para tensão relacionada ao trabalho 11 questões. As questões foram distribuídas de forma não sequencial, para evitar algum viés na hora de o respondente indicar sua resposta.

O questionário foi entregue aos coordenadores por meio da diretoria de cada área, administrativa, enfermagem e hotelaria, e solicitada sua devolução em no máximo 5 dias. Do total de 55 questionários entregues, retornaram 46 respondidos. Dos 9 questionários de coordenadores que não foram devolvidos, 3 coordenadores não responderam o questionário, e 6 estavam em período de férias. Os questionários respondidos representam 83,6\% dos questionários enviados. A aplicação do questionário ocorreu no mês de novembro de 2011. Os coordenadores que responderam ao questionário caracterizam-se por profissionais de nível superior das mais diversas áreas de formação como administração, ciências contábeis, assistência social, nutrição, psicologia e principalmente enfermagem.

Após receber os questionários, procedeu-se a tabulação dos dados, que consistiu num primeiro momento a reunião das questões que tratavam de cada uma das variáveis, para em seguida normalizar as respostas, possibilitando assim a análise estatística dos dados.

Apresentam-se na Tabela 1, as estatísticas descritivas das variáveis do estudo.

Tabela 1: Estatística descritiva e Alpha de Cronbach

\begin{tabular}{|c|c|c|c|c|c|c|c|}
\hline \multirow[t]{2}{*}{ Variáveis } & \multirow[t]{2}{*}{ Média } & \multirow[t]{2}{*}{ Mediana } & \multirow{2}{*}{$\begin{array}{l}\text { Desvio } \\
\text { padrão }\end{array}$} & \multirow{2}{*}{$\begin{array}{l}\text { Série } \\
\text { Atual }\end{array}$} & \multicolumn{2}{|c|}{ Série } & \multirow{2}{*}{$\begin{array}{l}\text { Alpha de } \\
\text { Cronbach }\end{array}$} \\
\hline & & & & & Min & Máx & \\
\hline Ênfase & 32,86 & 13,00 & 34,576 & 84 & 1 & 85 & $\mathrm{n} / \mathrm{a}$ \\
\hline Participação & 39,43 & 20,00 & 33,206 & 85 & 2 & 87 & 0,84 \\
\hline Confiança & 26,29 & 11,00 & 24,547 & 56 & 5 & 61 & 0,83 \\
\hline Tensão & 6,57 & 6 & 5,473 & 16 & 1 & 17 & 0,63 \\
\hline
\end{tabular}

Fonte: dados da pesquisa.

A próxima seção é dedicada à analise dos resultados, onde apresentam-se os resultados encontrados na pesquisa.

\section{Análise dos Resultados}

As principais relações que foram testadas no estudo, envolvem efeitos indiretos. Por esta razão, a análise do caminho é a técnica apropriada para investigar esses efeitos, pois Hair et al (2005) ensinam que a quantia de correlação é atribuída a cada efeito, a cada variável. A Tabela 2 apresenta as correlações entre as variáveis analisadas. Os resultados indicam que as correlações entre as variáveis são significativas ao nível de 0,01 .

Tabela 2: Correlação entre variáveis

\begin{tabular}{lccc}
\hline & Participação & Confiança & Tensão \\
\hline Ênfase & $0,987(*)$ & $0,982(*)$ & $-0,546\left(^{*}\right)$ \\
Participação & & $0,974(*)$ & $-0,482\left(^{*}\right)$ \\
Confiança & & & $-0,429\left(^{*}\right)$ \\
\hline
\end{tabular}

* Correlação significativa ao nível de 0.01 .

Para testar as hipóteses do estudo, foi necessário calcular inicialmente o coeficiente de caminho de cada variável, cujos dados são apresentados na Tabela 3.

Tabela 3: Resultado análise de caminho

\begin{tabular}{cccc}
\hline Variável dependente & Variável independente & Coeficiente Caminho & t-value \\
\hline Participação & Ênfase & 0,948 & 13,603 \\
Confiança & Ênfase & 0,697 & 11,792 \\
& Participação & 0,720 & 9,560 \\
Tensão & Ênfase & $-0,086$ & $-0,546$ \\
& Participação & $-0,079$ & $-1,231$ \\
& Confiança & $-0,096$ & $-1,063$ \\
\hline
\end{tabular}

A hipótese H1 afirma que existe uma relação indireta entre a ênfase no orçamento e tensão relacionada com o trabalho através da variável confiança, e a hipótese $\mathrm{H} 2$ afirma que existe uma relação indireta entre a ênfase no orçamento e tensão relacionada com o trabalho através da variável participação. 
Portanto, para medir os efeitos indiretos da ênfase no orçamento sobre a tensão relacionada ao trabalho, através das duas variáveis, compomos os caminhos vistos na Tabela 4.

Tabela 4: Efeitos indiretos da ênfase no orçamento sobre a tensão relacionada ao trabalho

\begin{tabular}{lllr}
\hline & Variáveis & Coeficientes & Efeito \\
\hline Caminho (1) & Ênfase - participação - tensão & $0,948 \times-0,079$ & $=-0,075$ \\
Caminho (2) & Ênfase - participação - confiança - tensão & $0,948 \times 0,72 \times-0,096$ & $=-0,065$ \\
Caminho (3) & Enfase - confiança - tensão & $0,697 \times-0,096$ & $=-0,067$ \\
& Total efeito indireto & & $=-0,207$ \\
\hline
\end{tabular}

Percebeu-se que o caminho (1) indica o efeito indireto exclusivamente via participação no orçamento, representado por -0,075. Os caminhos (2) e (3) indicam o efeito indireto através da confiança, de -0,132.

Com base neste resultado, pode-se afirmar, em relação à hipótese H1 que a ênfase no orçamento tem um efeito indireto sobre a tensão relacionada ao trabalho através da confiança e é confirmada através dos caminhos 2 e 3 , com um resultado de $-0,132$. E com relação à hipótese $\mathrm{H} 2$, é possível afirmar que a ênfase no orçamento tem um efeito indireto sobre a tensão relacionada ao trabalho através da participação orçamentária, é confirmada através do caminho 1 , com um resultado de $-0,075$.

A hipótese H3 afirma que existe uma relação indireta entre a participação no orçamento e tensão relacionada com o trabalho através da confiança. Para testar a hipótese, o efeito indireto é composto dos seguintes caminhos, calculados com base nos valores dos coeficientes de caminho verificados na Tabela 5.

Tabela 5: Efeito indireto entre participação no orçamento e tensão relacionada ao trabalho através da confiança

\begin{tabular}{lllr}
\hline & Variáveis & Coeficientes & Efeitos \\
\hline Caminho (4) & Participação - confiança - tensão & $0,720 \times-0,096$ & $=-0,069$ \\
Caminho (5) & Participação - ênfase - tensão & $0,948 \times-0,085$ & $=-0,081$ \\
Caminho (6) & Participação - ênfase - confiança - tensão & $0,948 \times 0,697 \times-0,096$ & $=-0,063$ \\
& Total efeito indireto & & $=-0,213$ \\
\hline
\end{tabular}

Percebeu-se que o caminho (4) indica o efeito indireto exclusivamente via confiança, representado pelo valor de $-0,069$. Os caminhos (5) e (6) indicam um efeito total espúrio de - 0,144 . De acordo com o estudo base de Lau e Buckland (2001), o efeito indireto via confiança está em excesso, portanto, H3 é suportada.

Com base nestes resultados, pode-se afirmar, em relação à hipótese H3 que a participação orçamentária tem um efeito indireto sobre a tensão relacionada ao trabalho através da confiança, é confirmada através do caminho (4).

Apresenta-se a seguir a Tabela 6, que mostra um resumo da decomposição das correlações para mensurar os efeitos diretos, indiretos e espúrios.

Tabela 6: Decomposição das correlações observadas

\begin{tabular}{lcccc}
\hline \multicolumn{1}{c}{ Relações } & Correlação Observada & Efeito Direto & Efeito Indireto & Efeito Espúrio \\
\hline Ênfase/Tensão & $-0,546$ & $-0,086$ & $-0,460$ & \\
Ênfase/Participação & 0,987 & 0,948 & & 0,039 \\
Participação/Confiança & 0,974 & 0,720 & & 0,254 \\
Ênfase/Confiança & 0,982 & 0,697 & 0,285 & \\
Confiança/Tensão & $-0,429$ & $-0,096$ & & $-0,333$ \\
Participação/Tensão & $-0,482$ & $-0,079$ & $-0,403$ & \\
\hline
\end{tabular}

O modelo proposto inicialmente por Lau e Buckland (2001), apropriados os resultados encontrados é demonstrado na Figura 3, demonstrando através de quais caminhos as hipóteses foram testadas.

Figura 3: Demonstração do resultado da pesquisa através da análise de caminhos

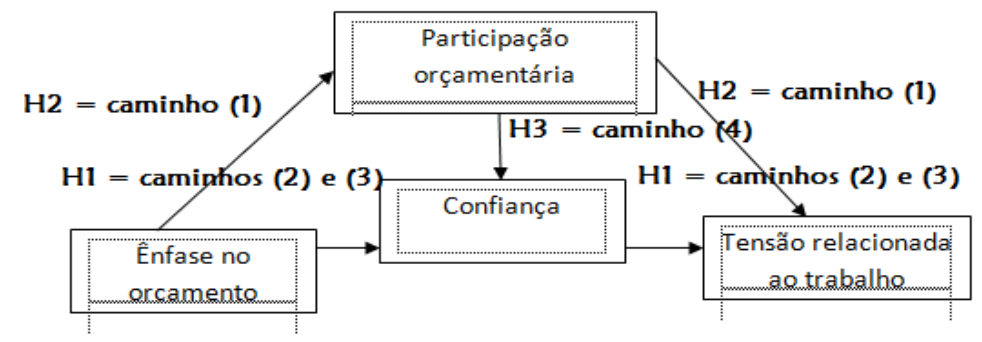

Fonte: Adaptado de Lau e Buckland (2001). 
Com base nos resultados encontrados, pode-se afirmar que os efeitos das variáveis, mesmo quando aplicados a uma organização filantrópica, não diferem dos resultados observados em estudos realizados em empresas de capital aberto e finalidade lucrativa.

\section{Considerações Finais}

Esta pesquisa procurou aplicar a mesma metodologia utilizada no estudo de Lau e Buckland (2001), realizado junto a gestores do setor privado lucrativo, para identificar se houvesse diferença em relação aos gestores deste tipo de organização, com finalidade lucrativa, de uma instituição filantrópica, sem fim lucrativo.

Para tanto, foram utilizadas as mesmas variáveis e a mesma forma de coleta e análise de dados, com a incorporação dos efeitos da confiança em relação à participação orçamentária, ênfase de orçamento e tensão relacionada ao trabalho.

Os resultados encontrados no estudo base e na presente pesquisa suportam as hipóteses desenvolvidas e, portanto, fornecem uma evidência adicional para ajudar a explicar a complexa relação entre estilos de avaliação do comportamento de subordinados.

Percebeu-se que os resultados foram semelhantes entre os dois estudos. Assim esta pesquisa juntamente com os resultados de outros trabalhos, fornece uma massa de conhecimentos, que tem por objetivo auxiliar na compreensão dos fatores envolvidos, independente do tipo de instituição pesquisada.

\section{Referências}

BROWNELL, P.; HIRST, M. Reliance on Accounting Information, Budgetary participation, and task uncertatinty: tests os a three-way interaction. Journal of Accounting Research, vol. 24, $\mathrm{n}^{\circ} 2$ (Autumn, 1986), pp.241-249.

FISCHER, R. M.; NOVELLI, J. G. N. Confiança como fator de redução da vulnerabilidade humana no ambiente de trabalho. RAE, n. 67, Abr/Jun 2008.

GIL, A. C. Como elaborar Projetos de Pesquisa. 4. Ed. 8 reimpr. São Paulo: Atlas, 2006.

HAIR, J. F.; ANDERSON, R. E.; TATHAM, R . L.; BLACK, W. C. Análise multivariada de dados. 5.ed. Porto Alegre: Bookmann, 2005.

HOFSTEDE, G. The cultural relativity of organizational practices and theories. Journal of International Business Studies. Fall 1983.

HOPWOOD, A. G. An empirical Study of the role of accounting data in performance evaluation. Journal of Accountig Research, Vol. 10, Empirical research in Accounting: selectedstudies 1972, pp; 156-182.

JOSHI, P.L.; AL-MUDHAKI, J.; BREMSER, W.G. Corporate budget planning, control and performance evaluation in Bahrain. Managerial Auditing Journal, 18/9, 2003, 737-750.

JUNQUEIRA, E.; OYADOMARI J. C. T.; MORAES, R. O. Reservas orçamentárias: um ensaio sobre fatores que levam à sua constituição. Revista: Contexto, Porto Alegre, v.10, ${ }^{\circ}$ 17, p.31-42, ISSN (Online): 2175$87511^{\circ}$ semestre 2010.

LAU, C.M.; LOW, L.C.; EGGLETON, I. R. The interactive effects of budget emphasis, participation and task difficulty on managerial performance: a cross-cultural study. Accounting, Auditing \& Accountability Journal. Vol. 10 n 2, 1997, pp.175-197.

LAU, C. M.; BUCKLAND, C. Budgeting - the role of trust and participation: a research note. ABACUS, vol. $37, \mathrm{n}^{\mathrm{o}} 3,2001$.

MILANI, K.; The Relationship of Participation in Budget-Setting to Industrial Supervisor Performance and Attitudes: A Field Study. The Accounting Review, Vol. 50, No. 2 (Apr., 1975), pp. 274-284.Published by: American Accounting Association Stable.

NASCIMENTO, A. R. Avaliação do orçamento como Instrumento de controle de gestão: um estudo de caso em uma indústria de fertilizantes. XII SIMPEP - Bauru, SP, 06 a 08 de novembro de 2006.

NASCIMENTO, A. R.; RIBEIRO, D. C.; JUNQUEIRA, E. R. Estado da Arte da abordagem comportamental da contabilidade gerencial: análise das pesquisas internacionais. Disponível em www.congressousp.fipecafi.org/artigos82008. acesso em 20/10/2011. 
NOURI, H.; PARKER, R.J. The relationship between budget participation and job performance: the roles of budget adequacy and organizational commitment. Accounting, Organizations and Society, vol. 23, $n^{\circ} 5 / 6$, pp.467-483, 1998.

NOVELLI, J. G. N.; FISCHER, R .M.; MAZZON, J. A. Fatores de confiança interpessoal no ambiente de trabalho. R. Adm., São Paulo, v.41, n.4, p.442-452, out/nov/dez. 2006.

OTLEY, D. T. Budget use and managerial performance. Journal of Accounting Research, vol. 16, $\mathrm{n}^{\circ} 1$ (spring 1978), pp.122-149.

RAUPP, F.M.; BEUREN, I.M. Como elaborar trabalho monográficos em contabilidade: teoria e prática. São Paulo: Atlas, 2003.

ROSS, A. Job related tension, budget emphasis and uncertainty: a research note. Management Accounting Research, 1995, 6, 1-11.

ZAND, D.E. Trust and Managerial Problem Solving. Administrative Science Quarterly, vol. 17, $\mathrm{n}^{\mathrm{o}} 2$ (jun, 1972), pp.229-239. 